\title{
Progress and management of denosumab- related severe osteonecrosis of the entire lower jaw- A case report
}

\author{
Tomic $\mathbf{J}^{*}$, Jakse $\mathrm{N}^{2}$ and Acham $\mathrm{S}^{3}$ \\ ${ }^{1}$ Division of Oral and Maxillofacial Surgery, Department of Dental Medicine and Oral Health, Medical University Graz, Graz, Austria \\ ${ }^{2}$ Interim Head of the Division of Oral and Maxillofacial Surgery, Head of the Division of Oral Surgery and Orthodontics, Department of Dental Medicine and Oral \\ Health, Medical University of Graz, Graz, Austria \\ ${ }^{3}$ Division of Oral Surgery and Orthodontics, Department of Dental Medicine and Oral Health, Medical University Graz, Graz, Austria
}

\begin{abstract}
Symptomatic patients with advanced stages of medication - related osteonecrosis (MRONJ) may require immediate aggressive surgical treatment but are often susceptible to common antibiotics. The case of a 71-year-old man with severe osteonecrosis of the entire lower jaw demonstrated good response to systemic antibiotic therapy. Operative therapy was directed at resecting the remaining local necrotic bone. Additionally, multiple skeletal- related events (SREs) occurred after antiresorptive treatment break. This case is used to highlight the long- term conservative treatment for the improvement and limitation of invasive procedures in cases with severe MRONJ. There is no strong evidence in the oral and maxillofacial surgery literature regarding the optimal procedural strategy and antiresorptive treatment break.
\end{abstract}

\section{Introduction}

The incidence of malignant tumors in Austria was 471 per 100,000 patients in 2015 and $20 \%$ out of those were affected by advanced malignoma [1,2].

Many patients with advanced malignant tumors are in need of long - term management of metastatic bone disease which is a common cause of cancer - related pain. Bone metastases can affect the axial skeleton. Skeletal morbidity adversely affects oncologic patients who experience poor quality of life due to skeletal- related events (SREs) such as pathological fracture and spinal cord compression. Antiresorptive therapy (ART) can reduce skeletal morbidity in combination with analgesic. SRE may rebound when antiresorptive treatment of lytic lesions is interrupted whereas temporary suspension of antiresorptive medication may preclude the development of osteonecrosis of the jaw (ONJ) [3]. The risk of ONJ in patients with metastatic bone disease from solid tumors exposed to antiresorptive agents is 100 times higher than in oncologic patients treated with placebo [4].

The diagnosis of osteonecrosis of the jaw (ONJ) involves a good history, clinical examination and radiographic analysis. Medicationrelated osteonecrosis (MRONJ) is a severe adverse drug reaction linked to various drugs and may occur many years after interrupting drug therapy. Clinical manifestations include non healing bone exposure, loosening of teeth, intraoral or extraoral fistula and pathologic fracture. Risk factors include use of denture, poor oral hygiene, infection (periodontitis), oral treatments (tooth extraction), steroid therapy and underlying diseases like diabetes, anemia, dialysis, osteomalacia, hypocalcemia, rheumatoid arthritis and immunosuppression. A history of radiotherapy or metastatic disease of the jaws should be ruled out prior to diagnosis of MRONJ [4].

We report a case of an elderly man who developed a stage 3 medication- related osteonecrosis (MRONJ) that showed nearcomplete regression after conservative therapy.

\section{Case Report}

A 71-year-old man with advanced malignant melanoma of unknown primary presented with pain and paresthesia to our outpatient clinic at the Division of Oral Surgery and Orthodontics. The patient received longterm antiresorptive therapy (ART) with monthly injections of denosumab. Additionally chemotherapy and radiation therapy for metastatic invasion of cervical and thoracical spine were given to our patient. Our patient showed poor general condition, pain and anaesthesia of the mental region shortly after initial presentation. An oral examination revealed several fistulas on the alveolar ridge in the anterior mandible with evidence of infection and drainage of pus. The Panoramic X- ray showed a diffuse bone alteration and cortical erosion of the entire corpus mandibulae (Figure 1, Panel A), which was contributory to the diagnosis of medication- related osteonecrosis (MRONJ). An adverse effect of radiation therapy on the jaws was definitely ruled out by our radiotherapists. As our patient initially rejected surgical therapy we followed a conservative management including a prolonged course of oral amoxicillin and clavulanic acid, pain medication, proton-pump inhibitors (PPIs) and strict avoidance of partial denture. In agreement with oncologists ART was paused. Additional irrigations of local fistulas and supportive low level laser therapy (LLLT) were applied at least twice a week. On follow- up 1 year after admission we observed resolution of the extensive radiographic

${ }^{*}$ Correspondence to: Josip Tomic, Division of Oral Surgery and Orthodontics, Department of Dental Medicine and Oral Health, Med-ical University Graz, Austria, E-mail: josip.tomic@medunigraz.at

Key words: medication- related osteonecrosis of the jaw (MRONJ), antiresorptive drugs, bisphosphonates, denosumab, skeletal-related events (SREs)

Received: March 13, 2019; Accepted: March 21, 2019; Published: March 23, 2019 
abnormalties (Figure 1, Panel B) and our patient's general medical condition improved. But our patient had one persistent fistula without purulent drainage (Figure 2, Panel A) and paresthesia of the right premolar mandibular region. After having informed consent decortication and surgical debridement of infected soft and bone tissues were performed including the extraction of involved teeth \# 26 and \# 27 (Figure 2, Panel B and C). Several biopsy specimens were obtained. Histologic analysis of surgical bone samplings revealed evidence of chronic tissue inflammation without sulfur granules of commensal actinomyces.

Our patient showed complete healing of exposed bone with no further symptoms on follow- up one year after surgery (Figure 2, Panel D). Even though the re- introduction of ART was advised after reaching soft tissue healing, our patient refused resumption of antiresroptive medication. Another SRE (stable fracture of vertebral body T 7) was reported soon after the last follow- up with 16 months discontinuation of denosumab therapy.

\section{Discussion}

Our patient case showed near complete regression under conservative treatment in combination with antiresorptive drugholiday and close monitoring (Figure 1, Panel A and B). Surgical treatment of persistent non- healing bone lesion was successful.
Our patient had a history of multiple organ metastases upon admission. We ruled out metastatic infiltration of the lower jaw after biopsy and histopathologic examination. According to literature, metastases to the jaws are uncommon and comprise $1 \%$ of oral malignant tumors. Clinical findings can be variable. Most metastases are located in one site of mandibular molar region and radiographic findings show a single osteolytic lesion [5].

Palliative radiation therapy was delivered in our patient for metastatic bone alteration of the spine. While dose distribution is limited to the tumor lesion alone, we discussed whether low dose radiotherapy (RT) of upper cervical spine (C 1- C 3) in the patient presented here was related to the development of rapid onset osteonecrosis of the jaw (ONJ). However, osteoradionecrosis (ORN) develops in patients with high therapeutic dose (not less than 60 Gy) RT for oral cavity and oropharynx cancers after mandibular surgery [6].

Some authors found the indication for antibiotic treatment to be speculative at best, since denosumab- related osteonecrosis is a vascular mediated condition. Surgical treatment includes debridement, sequestrectomy, curettage, bone resection and reconstruction of diseased jawbone. However, it is still unclear which surgical modality shows the lowest recurrence rate of medication- related osteonecrosis
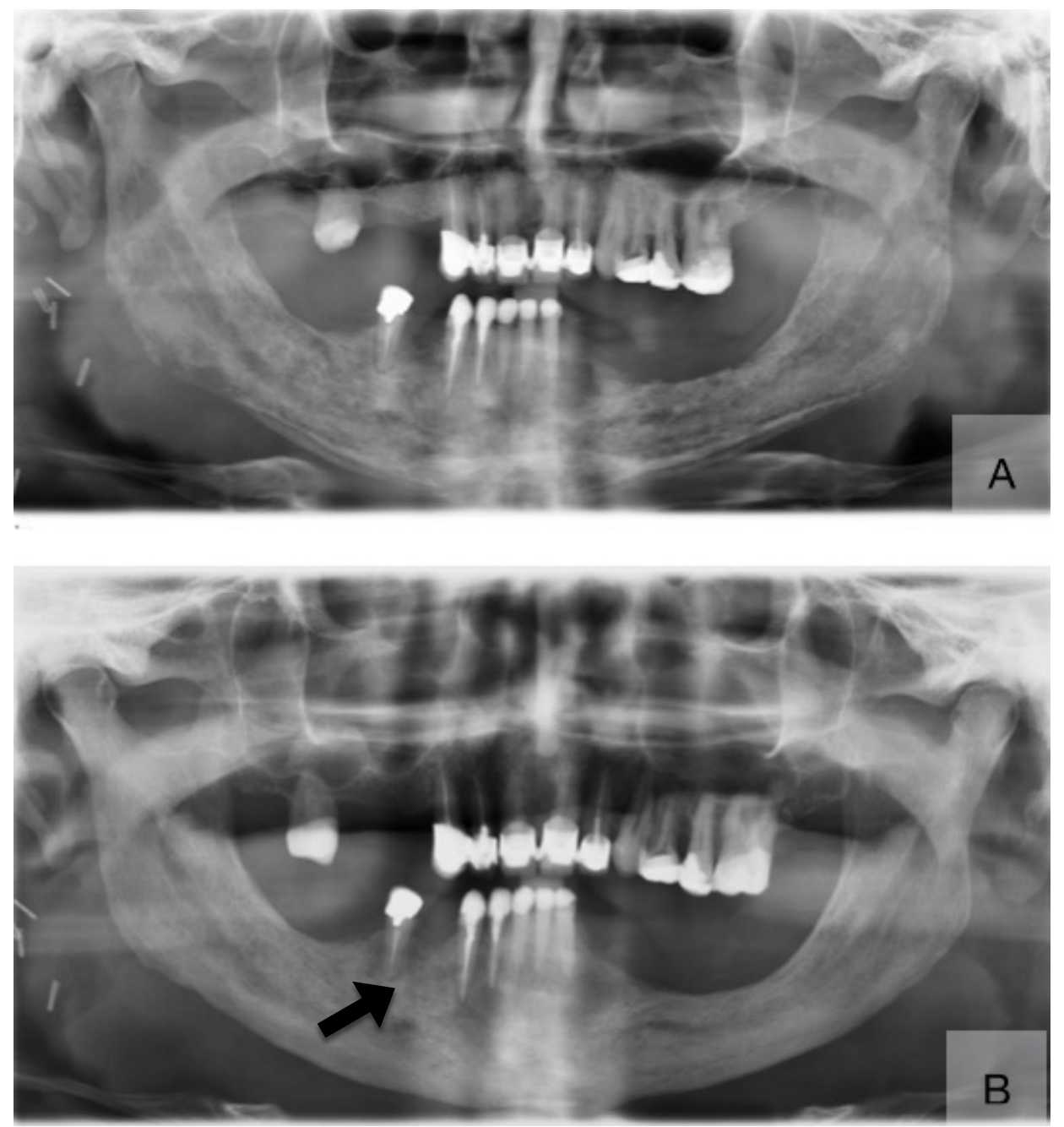

Figure 1. Panoramic imaging obtained on admission with the typical aspect of mixed radiologic density of bone and periosteal formation of mandibular inferior cortical bone before surgery (Panel A). Resolution of all radiologic signs but one residual necrotic area (arrow) on follow up remained four months before operative therapy (Panel B) 


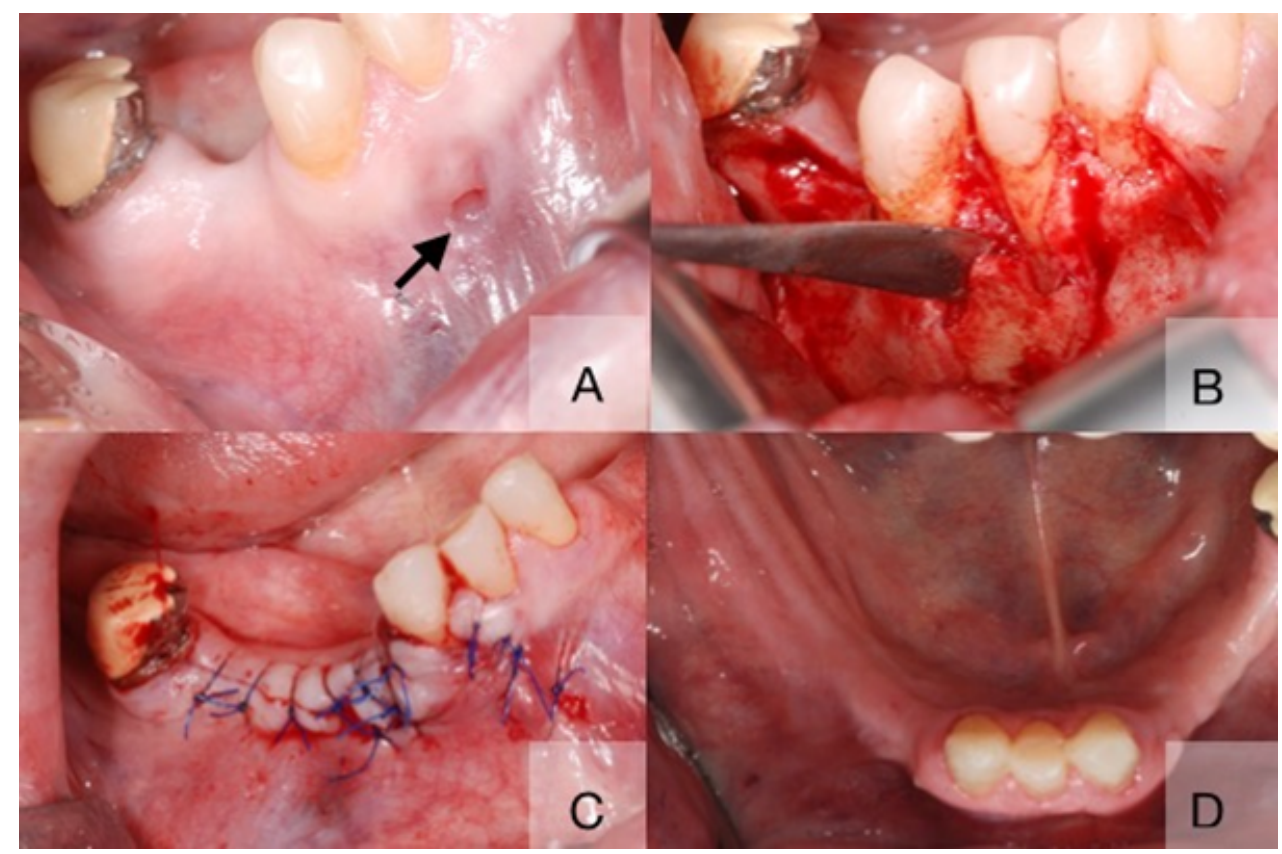

Figure 2. Segment of necrotic bone could be probed through an intraoral fistula (Panel A, arrow) and was mobile (Panel B). The surgical procedure consisted of resection of necrotic parts and extraction of \# 26 and \# 27 (Panel C) and dense, multi- row wound closure (Panel C). Stable soft tissue situation two months after surgery (Panel D)

of the jaw (MRONJ) [4]. A surgical approach is often favored over conservative treatment after careful selection of patients. The last position paper of the American Association of Oral and Maxillofacial Surgeons (AAOMS) highlights that surgical treatment is used for high stages of MRONJ [4].

Conservative treatment strategies consist of pain medication, antibiotics (e.g. penicillin group of antibiotics), antibacterial mouth rinse (e.g. chlorhexidine $0.12 \%$ ), patient education (e.g. dental care, fluoride application, denture stability) and regular clinical follow- up [4]. Some authors favored conservative treatment over surgery. In their report they have described that the administration of intravenous penicillin for a prolonged period (4 to 6 weeks) may be indicated in severe cases of MRONJ [7].

In addition the suspension of Denosumab is controversial since there is little evidence to establish the role of antiresorptive or antiangiogenic drug interruption. Our patient has experienced multiple skeletal- related events (SREs) after discontinuation of Denosumab. Antiresorptive therapy was described to reduce single and multiple SREs in a metaanalysis that identified an increased risk of subsequent events in patients with SRE when compared to patients without SRE [8]. Drug interruption may be suggested in patients with osteoporosis after the patients have improved in bone density.

An international task force recommended a treatment break of ART in absence or presence of ONJ until the occurrence of soft tissue healing [9]. More recent literature elucidated an increased fracture risk after cessation of denosumab as evident in the case presented here [10]. Discontinuing antiresorptive drugs in the presence of ONJ or in patients with high risk of ONJ (e.g. diabetes, glucocorticoids, immune deficiencies, smoking) may be indicated until wound closure is obtained. However, the American Dental Association's (ADA) expert panel does not support "drug holidays" [4]. These issues underscore the need for additional studies on suspension of denosumab to prevent bone loss and fractures.

\section{Funding}

This research paper will be funded by the International Postgraduate Summer School in Dentistry 2019, Medical University of Graz, Austria.

\section{Competing interests}

None

\section{Ethical approval}

Not required

\section{Patient consent}

The patient signed a patient release of information form to authorize release of the patient's clinical photographs.

\section{References}

1. http://www.statistik.at/web_de/statistiken/menschen_und_gesellschaft/gesundheit/ krebserkrankungen/krebs_im_ueberblick/index.html

2. Coleman RE (2006) Clinical features of metastatic bone disease and risk of skeletal morbidity. Clin Cancer Res 12: 6243s-6249s. [Crossref]

3. Niimi R, Kono T, Nishihara A, Hasegawa M, Kono T, et al. (2018) Rebound-associated vertebral fractures af-ter discontinuation of denosumab for the treatment of maxillitis. Osteoporos Int 29: 769-772. [Crossref]

4. Ruggiero SL, Dodson TB, Fantasia J, Goodday R, Aghaloo T, et al. (2014) American Association of Oral and Maxillofacial Surgeons position paper on medication-related osteonecrosis of the jaw--2014 update. J Oral Maxillofac Surg 72: 1938-1956. [Crossref]

5. Kumar G, Manjunatha B (2013) Metastatic tumors to the jaws and oral cavity. J Oral Maxillofac Pathol 17: 71-75. [Crossref]

6. Felice FD, Piccioli A, Musio D, Tombolini V (2017) The role of radiation therapy in bone metas-tases management. Oncotarget 8: 25691-25699. [Crossref]

7. Lazarovici TS, Yahalom R, Taicher S, Elad S, Hardan I, et al. (2009) Bisphosphonaterelated osteonecrosis of the jaws: a single-center study of 101 patients. J Oral Maxillofac Surg 67: 850-855. [Crossref]

8. Lipton A, Fizazi K, Stopeck AT, Henry DH, Brown JE, et al. (2012) Superiority of denosumab to zoledronic acid for prevention of skeletal-related events: a combined analysis of 3 pivotal, randomised, phase 3 trials. Eur J Cancer 48: 3082-3092. [Crossref] 
Tomic J (2019) Progress and management of denosumab- related severe osteonecrosis of the entire lower jaw- A case report

9. Khan AA, Morrison A, Hanley DA, Felsenberg D, McCauley LK, et al. (2015) Diagnosis and management of osteonecrosis of the jaw: a systematic review and international consensus. J Bone Miner Res 30: 3-23. [Crossref]
10. Tsourdi E, Langdahl B, Cohen-Solal M, Aubry-Rozier B, Eriksen EF, et al. (2017) Discontinuation of Denosumab therapy for osteoporosis: A systematic review and position statement by ECTS. Bone 105: 11-17. [Crossref]

Copyright: (C2019 Tomic J. This is an open-access article distributed under the terms of the Creative Commons Attribution License, which permits unrestricted use, distribution, and reproduction in any medium, provided the original author and source are credited. 